\title{
The Cryptographic Power of Random Selection
}

\author{
Matthias Krause and Matthias Hamann \\ Theoretical Computer Science \\ University of Mannheim \\ Mannheim, Germany
}

\begin{abstract}
The principle of random selection and the principle of adding biased noise are new paradigms used in several recent papers for constructing lightweight RFID authentication protocols. The cryptographic power of adding biased noise can be characterized by the hardness of the intensively studied Learning Parity with Noise (LPN) Problem. In analogy to this, we identify a corresponding learning problem for random selection and study its complexity. Given $L$ secret linear functions $f_{1}, \ldots, f_{L}:\{0,1\}^{n} \longrightarrow\{0,1\}^{a}$, RandomSelect $(L, n, a)$ denotes the problem of learning $f_{1}, \ldots, f_{L}$ from values $\left(u, f_{l}(u)\right)$, where the secret indices $l \in\{1, \ldots, L\}$ and the inputs $u \in\{0,1\}^{n}$ are randomly chosen by an oracle. We take an algebraic attack approach to design a nontrivial learning algorithm for this problem, where the running time is dominated by the time needed to solve full-rank systems of linear equations over $O\left(n^{L}\right)$ unknowns. In addition to the mathematical findings relating correctness and average running time of the suggested algorithm, we also provide an experimental assessment of our results.
\end{abstract}

Keywords: Lightweight Cryptography, Algebraic Attacks, Algorithmic Learning, Foundations and Complexity Theory.

\section{Introduction}

The very limited computational resources available in technical devices like RFID (radio frequency identification) tags implied an intensive search for lightweight authentication protocols in recent years. Standard block encryption functions like Triple-DES or AES seem to be not suited for such protocols largely because the amount of hardware to implement and the energy consumption to perform these operations is too high (see, e.g., 7] or [17] for more information on this topic).

This situation initiated two lines of research. The first resulted in proposals for new lightweight block encryption functions like PRESENT [4, KATAN and KTANTAN [10 by use of which standard block cipher-based authentication protocols can be made lightweight, too. A second line, and this line we follow in the paper, is to look for new cryptographic paradigms which allow for designing new symmetric lightweight authentication protocols. The two main suggestions discussed so far in the relevant literature are the principle of random selection and the principle of adding biased noise. 
The principle of adding biased noise to the output of a linear basis function underlies the HB-protocol, originally proposed by Hopper and Blum [16] and later improved to $\mathrm{HB}^{+}$by Juels and Weis [17, as well as its variants $\mathrm{HB}^{\#}$ and Trusted-HB (see [13] and [6], respectively). The protocols of the HB-family are provably secure against passive attacks with respect to the Learning Parity with Noise Conjecture but the problem to design HB-like protocols which are secure against active adversaries seems to be still unsolved (see, e.g., [14, [21, [12]).

The principle of random selection underlies, e.g., the CKK-protocols of Cichon, Klonowski, and Kutyłowski [7] as well as the $F_{f}$-protocols in [3] and the Linear Protocols in [18. It can be described as follows.

Suppose that the verifier Alice and the prover Bob run a challenge-response authentication protocol which uses a lightweight symmetric encryption operation $E:\{0,1\}^{n} \times \mathcal{K} \longrightarrow\{0,1\}^{m}$ of block length $n$, where $\mathcal{K}$ denotes an appropriate key space. Suppose further that $E$ is weak in the sense that a passive adversary can efficiently compute the secret key $K \in \mathcal{K}$ from samples of the form $\left(u, E_{K}(u)\right)$. This is obviously the case if $E$ is linear.

Random selection denotes a method for compensating the weakness of $E$ by using the following mode of operation. Instead of holding a single $K \in \mathcal{K}$, Alice and Bob share a collection $K_{1}, \ldots, K_{L}$ of keys from $\mathcal{K}$ as their common secret information, where $L>1$ is a small constant. Upon receiving a challenge $u \in$ $\{0,1\}^{n}$ from Alice, Bob chooses a random index $l \in\{1, \ldots, L\}$ and outputs the response $y=E\left(u, K_{l}\right)$. The verification of $y$ with respect to $u$ can be efficiently done by computing $E_{K_{l}}^{-1}(y)$ for all $l=1, \ldots, L$.

The main problem this paper is devoted to is to determine the level of security which can be reached by applying this principle of random selection.

Note that the protocols introduced in [7], [3], and [18] are based on random selection of $G F(2)$-linear functions. The choice of linear basis functions is motivated by the fact that they can be implemented efficiently in hardware and have desirable pseudo-random properties with respect to a wide range of important statistical tests.

It is quite obvious that, with respect to passive adversaries, the security of protocols which use random selection of linear functions can be bounded from above by the complexity of the following learning problem referred to as RandomSelect $(L, n, a)$ : Learn $G F(2)$-linear functions $f_{1}, \ldots, f_{L}:\{0,1\}^{n} \longrightarrow$ $\{0,1\}^{a}$ from values $\left(u, f_{l}(u)\right)$, where the secret indices $l \in\{1, \ldots, L\}$ and the inputs $u \in\{0,1\}^{n}$ are randomly chosen by an oracle. In order to illustrate this notion, we sketch in appendix B how an efficient learning algorithm for RandomSelect $(L, n, a)$ can be used for attacking the linear $(n, k, L)^{+}$-protocol described by Krause and Stegemann [18.

In this paper, we present an algebraic attack approach for solving the above learning problem RandomSelect $(L, n, a)$. The running time of our algorithm is dominated by the effort necessary to solve a full-rank system of linear equations of $O\left(n^{L}\right)$ unknowns over the field $G F\left(2^{a}\right)$. Note that trivial approaches for solving RandomSelect $(L, n, a)$ lead to a running time exponential in $n$. 
In recent years, people from cryptography as well as from complexity and coding theory devoted much interest to the solution of learning problems around linear structures. Prominent examples in the context of lightweight cryptography are the works by Goldreich and Levin [15], Regev [22], and Arora and Ge [2. But all these results are rather connected to the Learning Parity with Noise Problem. To the best of our knowledge, there are currently no nontrivial results with respect to the particular problem of learning randomly selected linear functions, which is studied in the present paper.

We are strongly convinced that the complexity of RandomSelect also defines a lower bound on the security achievable by protocols using random selection of linear functions, e.g., the improved $(n, k, L)^{++}$-protocol in 18 . Thus, the running time of our algorithm hints at how the parameters $n, k$, and $L$ should be chosen in order to achieve an acceptable level of cryptographic security. Note that choosing $n=128$ and $L=8$ or $n=256$ and $L=4$, solving RandomSelect $(L, n, a)$ by means of our algorithm implies solving a system of around $2^{28}$ unknowns, which should be classified as sufficiently difficult in many practical situations.

The paper is organized as follows. In sections 2, 3, and 4, our learning algorithm, which conducts an algebraic attack in the spirit of [23], will be described in full detail. We represent the $L$ linear basis functions as assignments $A$ to a collection $X=\left(x_{i}^{l}\right)_{i=1, \ldots, n, l=1, \ldots, L}$ of variables taking values from the field $K=G F\left(2^{a}\right)$. We will then see that each example $\left(u, f_{l}(u)\right)$ induces a degree$L$ equation of a certain type in the $X$-variables, which allows for reducing the learning problem RandomSelect $(L, n, a)$ to the problem of solving a system of degree- $L$ equations over $K$. While, in general, the latter problem is known to be NP-hard, we can show an efficient way to solve this special kind of systems.

One specific problem of our approach is that, due to inherent symmetries of the degree- $L$ equations, we can never reach a system which has full linear rank with respect to the corresponding monomials. In fact, this is the main difference between our learning algorithm and the well-known algebraic attack approaches for cryptanalyzing LFSR-based keystream generators (see, e.g., [20], [8], [9], [1]).

We circumvent this problem by identifying an appropriate set $T(n, L)$ of basis polynomials of degree at most $L$ which allow to express the degree- $L$ equations as linear equations over $T(n, L)$. The choice of $T(n, L)$ will be justified by Theorem 2 saying that if $|K| \geq L$, then the system of linear equations over $T(n, L)$ induced by all possible examples has full rank $|T(n, L)|$. (Note that according to Theorem [1] this is not true if $|K|<L$.) Our experiments, which are presented in section 5. indicate that if $|K| \geq L$, then with probability close to one, the number of examples needed to get a full rank system over $T(n, L)$ exceeds $|T(n, L)|$ only by a small constant factor. This implies that the effort to compute the unique weak solution $t(A)=\left(t_{*}(A)\right)_{t_{*} \in T(n, L)}$ corresponding to the strong solution $A$ equals the time needed to solve a system of $|T(n, L)|$ linear equations over $K$.

But in contrast to the algebraic attacks in [20, 8], [9, [1, we still have to solve another nontrivial problem, namely, to compute the strong solution $A$, which identifies the secret functions $f_{1}, \ldots, f_{L}$, from the unique weak solution. An efficient way to do this will complete our learning algorithm for 
RandomSelect $(L, n, a)$ in section 4. Finally, we also provide an experimental evaluation of our estimates using the computer algebra system Magma [5] in section [5] and conclude this paper with a discussion of the obtained results as well as an outlook on potentially fruitful future work in section 6 .

\section{The Approach}

We fix positive integers $n, a, L$ and secret $G F(2)$-linear functions $f_{1}, \ldots, f_{L}$ : $\{0,1\}^{n} \longrightarrow\{0,1\}^{a}$. The learner seeks to deduce specifications of $f_{1}, \ldots, f_{L}$ from an oracle which outputs in each round an example $(u, w) \in\{0,1\}^{n} \times\{0,1\}^{a}$ in the following way. The oracle chooses independently and uniformly a random input $u \in_{U}\{0,1\}^{n}$, then chooses secretly a random index $l \in_{U}[L] 1$, computes $w=f_{l}(u)$ and outputs $(u, w)$.

It is easy to see that RandomSelect can be efficiently solved in the case $L=1$ by collecting examples $\left(u^{1}, w_{1}\right), \ldots,\left(u^{m}, w_{m}\right)$ until $\left\{u^{1}, \ldots, u^{m}\right\}$ contains a basis of $G F(2)^{n}$. The expected number of iterations until the above goal is reached can be approximated by $n+1.61$ (see, e.g., the appendix in [1]).

We will now treat the case $L>1$, which immediately yields a sharp rise in difficulty. First we need to introduce the notion of a pure basis.

Definition 1. Let us call a set $\mathcal{V}=\left\{\left(u^{1}, w_{1}\right), \ldots,\left(u^{n}, w_{n}\right)\right\}$ of $n$ examples a pure basis, if $\left\{u^{1}, \ldots, u^{n}\right\}$ is a basis of $G F(2)^{n}$ and there exists an index $l \in[L]$ such that $w_{i}=f_{l}\left(u^{i}\right)$ is satisfied for all $i=1, \ldots, n$.

Recalling our preliminary findings, we can easily infer that for $m \in L n+\Omega(1)$, a set of $m$ random examples contains such a pure basis with high probability. Moreover, note that for a given set $\tilde{\mathcal{V}}=\left\{\left(\tilde{u}^{1}, \tilde{w}_{1}\right), \ldots,\left(\tilde{u}^{n}, \tilde{w}_{n}\right)\right\}$ the pure basis property can be tested efficiently. The respective strategy makes use of the fact that in case of a random example $(u, w)$, where $u=\bigoplus_{i \in I} \tilde{u}^{i}$ and $I \subseteq[n]$, the probability $p$ that $w=\bigoplus_{i \in I} \tilde{w}_{i}$ holds is approximately $L^{-1}$ if $\tilde{\mathcal{V}}$ is pure and at most $(2 \cdot L)^{-1}$ otherwise. The latter estimate is based on the trivial observation that if $\tilde{\mathcal{V}}$ is not a pure basis, it contains at least one tuple $\left(\tilde{u}^{j}, \tilde{w}_{j}\right), j \in[n]$, which would have to be exchanged to make the set pure. As $j \in I$ holds true for half of all possible (but valid) examples, the probability that $w=\bigoplus_{i \in I} \tilde{w}_{i}$ is fulfilled although $\tilde{\mathcal{V}}$ is not pure can be bounded from above by $(2 \cdot L)^{-1}$.

However, it seems to be nontrivial to extract a pure basis from a set of $m \in$ $L n+\Omega(1)$ examples. Exhaustive search among all subsets of size $n$ yields a running time exponential in $n$. This can be shown easily by applying Stirling's formula 3 to the corresponding binomial coefficient $\left(\begin{array}{c}m \\ n\end{array}\right)$.

\footnotetext{
${ }^{1}$ For a positive integer $N$, we denote by $[N]$ the set $\{1, \ldots, N\}$.

${ }^{2}$ Let $B=\left\{v^{1}, \ldots, v^{n}\right\}$ denote a basis spanning the vector space $V$. It is a simple algebraic fact that every vector $v \in V$ has a unique representation $I \subseteq[n]$ over $B$, i.e., $v=\bigoplus_{i \in I} v^{i}$.

${ }^{3}$ Stirling's formula is an approximation for large factorials and commonly written $n ! \approx \sqrt{2 \pi n}\left(\frac{n}{e}\right)^{n}$.
} 
We exhibit the following alternative idea for solving RandomSelect $(L, n, a)$ for $L>1$. Let $e^{1}, \ldots, e^{n}$ denote the standard basis of the $G F(2)$-vector space $\{0,1\}^{n}$ and keep in mind that $\{0,1\}^{n}=G F(2)^{n} \subseteq K^{n}$, where $K$ denotes the field $G F\left(2^{a}\right)$. For all $i=1, \ldots, n$ and $l=1, \ldots, L$ let us denote by $x_{i}^{l}$ a variable over $K$ representing $f_{l}\left(e^{i}\right)$. Analogously, let $A$ denote the $(n \times L)$-matrix with coefficients in $K$ completely defined by $A_{i, l}=f_{l}\left(e^{i}\right)$. Henceforth, we will refer to $A$ as a strong solution of our learning problem, thereby indicating the fact that its coefficients fully characterize the underlying secret $G F(2)$-linear functions $f_{1}, \ldots, f_{L}$.

Observing an example $(u, w)$, where $u=\bigoplus_{i \in I} e^{i}$, the only thing we know is that there is some index $l \in[L]$ such that $w=\bigoplus_{i \in I} A_{i, l}$. This is equivalent to the statement that $A$ is a solution of the following degree- $L$ equation in the $x_{i}^{l}$-variables.

$$
\left(\bigoplus_{i \in I} x_{i}^{1} \oplus w\right) \cdot \ldots \cdot\left(\bigoplus_{i \in I} x_{i}^{L} \oplus w\right)=0 .
$$

Note that equation (11) can be rewritten as

$$
\bigoplus_{J \subseteq I, 1 \leq|J| \leq L^{\prime}} \bigoplus_{j=|J|}^{L} w^{L-j} t_{J, j}=w^{L}
$$

$L^{\prime}=\min \{L,|I|\}$, where the basis polynomials $t_{J, j}$ are defined as

$$
t_{J, j}=\bigoplus_{g,|\operatorname{dom}(g)|=j, i m(g)=J} m_{g}
$$

for all $J \subseteq[n], 1 \leq|J| \leq L$, and all $j,|J| \leq j \leq L$. The corresponding monomials $m_{g}$ are in turn defined as

$$
m_{g}=\prod_{l \in \operatorname{dom}(g)} x_{g(l)}^{l}
$$

for all partial mappings $g$ from $[L]$ to $[n]$, where $\operatorname{dom}(g)$ denotes the domain of $g$ and $i m(g)$ denotes its image.

Let $T(n, L)=\left\{t_{J, j}|J \subseteq[n], 1 \leq| J|\leq L| J \mid, \leq j \leq L\right\}$ denote the set of all basis polynomials $t_{J, j}$ which may appear as part of equation (2). Moreover, we define

$$
\Phi(a, b)=\sum_{i=0}^{b}\left(\begin{array}{l}
a \\
i
\end{array}\right)
$$

for integers $0 \leq b \leq a$ and write 


$$
\begin{aligned}
|T(n, L)| & =\sum_{j=1}^{L}\left(\begin{array}{l}
n \\
j
\end{array}\right)(L-j+1) \\
& =(L+1)(\Phi(n, L)-1)-\sum_{j=1}^{L} n\left(\begin{array}{c}
n-1 \\
j-1
\end{array}\right) \\
& =(L+1)(\Phi(n, L)-1)-n \Phi(n-1, L-1) .
\end{aligned}
$$

Consequently, each set of examples $\mathcal{V}=\left\{\left(u^{1}, w_{1}\right), \ldots,\left(u^{m}, w_{m}\right)\right\}$ yields a system of $m$ degree- $L$ equations in the $x_{i}^{l}$-variables, which can be written as $m K$ linear equations in the $t_{J, j}$-variables. In particular, the strong solution $A \in K^{n \times L}$ satisfies the relation

$$
M(\mathcal{V}) \circ t(A)=W(\mathcal{V})
$$

where

- $K^{n \times L}$ denotes the set of all $(n \times L)$-matrices with coefficients from $K$,

- $M(\mathcal{V})$ is an $(m \times|T(n, L)|)$-matrix built by the $m$ linear equations of type (2D) corresponding to the examples in $\mathcal{V}$,

- $W(\mathcal{V}) \in K^{m}$ is defined by $W(\mathcal{V})_{i}=w_{i}^{i}$ for all $i=1, \ldots, m$,

- $t(A) \in K^{T(n, L)}$ is defined by $t(A)=\left(t_{J, j}(A)\right)_{J \subseteq[n], 1 \leq|J| \leq L,|J| \leq j \leq L}$.

Note that in section 3, we will treat the special structure of $M(\mathcal{V})$ in further detail. Independently, it is a basic fact from linear algebra that if $M(\mathcal{V})$ has full column rank, then the linear system (4) has the unique solution $t(A)$, which we will call the weak solution.

Our learning algorithm proceeds as follows:

(1) Grow a set of examples $\mathcal{V}$ until $M(\mathcal{V})$ has full column $\operatorname{rank}|T(n, L)|$.

(2) Compute the unique solution $t(A)$ of system (4), i.e., the weak solution of our learning problem, by using an appropriate algorithm which solves systems of linear equations over $K$.

(3) Compute the strong solution $A$ from $t(A)$.

We discuss the correctness and running time of steps (11) and (2) in section 3 and an approach for step (3) in section 4 .

\section{On Computing a Weak Solution}

Let $n$ and $L$ be arbitrarily fixed such that $2 \leq L \leq n$ holds. Moreover, let $\mathcal{V} \subseteq\{0,1\}^{n} \times K$ denote a given set of examples obtained through linear functions

\footnotetext{
${ }^{4}$ Keep in mind that, unlike for the previously introduced $K$-variables $x_{s}^{1}, \ldots, x_{s}^{L}, s \in$ $[n]$, the superscripted $L$ in case of $w_{i}^{L}$ is not an index but an exponent. See, e.g., equation (2).
} 
$f_{1}, \ldots, f_{L}:\{0,1\}^{n} \longrightarrow K$, where $K=G F\left(2^{a}\right)$. By definition, for each tuple $(u, w) \in \mathcal{V}$, where $u=\bigoplus_{i \in I} e^{i}$ and $I \subseteq[n]$ denotes the unique representation of $u$ over the standard basis $e^{1}, \ldots, e^{n}$ of $\{0,1\}^{n}$, the relation $w=f_{l^{\prime}}(u)=$ $\bigoplus_{i \in I} f_{l^{\prime}}\left(e^{i}\right)$ is bound to hold for some $l^{\prime} \in[L]$. We denote by $K^{\text {min }} \subseteq K$ the subfield of $K$ generated by all values $f_{l}\left(e^{i}\right)$, where $l \in[L]$ and $i \in[n]$. Note that $w \in K^{\mathrm{min}}$ for all examples $(u, w)$ induced by $f_{1}, \ldots, f_{l}$.

In the following, we show that our learning algorithm is precluded from succeeding if the secret linear functions $f_{1}, \ldots, f_{L}$ happen to be of a certain type or if $K$ itself lacks in size.

Theorem 1. If $\left|K^{\mathrm{min}}\right|<L$, then the columns of $M(\mathcal{V})$ are linearly dependent for any set $\mathcal{V}$ of examples, i.e., a unique weak solution does not exist.

Proof: Let $n, K, L$, and $f_{1}, \ldots, f_{L}$ be arbitrarily fixed such that $2 \leq\left|K^{\mathrm{min}}\right|<$ $L \leq n$ holds and let $\mathcal{V}$ denote a corresponding set of examples. Obviously, for each tuple $(u, w) \in \mathcal{V}$, where $u=\bigoplus_{i \in I} e^{i}$ and $I \subseteq[n]$, the two cases $1 \in I$ and $1 \notin I$ can be differentiated.

If $1 \in I$ holds, then it follows straightforwardly from equation (2) that the coefficient with coordinates $(u, w)$ and $t_{\{1\},(L-1)}$ in $M(\mathcal{V})$ equals $w^{L-(L-1)}=$ $w^{1}$. Analogously, the coefficient with coordinates $(u, w)$ and $t_{\{1\},\left(L-\left|K^{\min }\right|\right)}$ in $M(\mathcal{V})$ equals $w^{L-\left(L-\left|K^{\mathrm{min}}\right|\right)}=w^{\left|K^{\mathrm{min}}\right|}$. Note that $t_{\{1\},\left(L-\left|K^{\mathrm{min}}\right|\right)}$ is a valid (and different) basis polynomial as

$$
|\{1\}|=1 \leq\left(L-\left|K^{\min }\right|\right) \leq(L-2)<(L-1)<L
$$

holds for $2 \leq\left|K^{\text {min }}\right|<L$. As $K^{\text {min }} \subseteq K$ is a finite field of characteristic 2, we can apply Lagrange's theorem and straightforwardly conclude that the relation $z^{1}=z^{\left|K^{\text {min }}\right|}$ holds for all $z \in K^{\text {min }}$ (including $0 \in K^{\text {min }}$ ). Hence, if $1 \in I$ holds for an example $(u, w)$, then in the corresponding row of $M(\mathcal{V})$ the two coefficients indexed by $t_{\{1\},(L-1)}$ and $t_{\{1\},\left(L-\left|K^{\min }\right|\right)}$ are always equal.

If $1 \notin I$ holds for an example $(u, w)$, then the coefficient with coordinates $(u, w)$ and $t_{\{1\},(L-1)}$ in $M(\mathcal{V})$ as well as the coefficient with coordinates $(u, w)$ and $t_{\{1\},\left(L-\left|K^{\mathrm{min}}\right|\right)}$ in $M(\mathcal{V})$ equals 0 .

Consequently, if $\left|K^{\text {min }}\right|<L$ holds, then the column of $M(\mathcal{V})$ indexed by $t_{\{1\},(L-1)}$ equals the column indexed by $t_{\{1\},(L-|K|)}$ for any set $\mathcal{V}$ of examples, i.e., $M(\mathcal{V})$ can never achieve full column rank.

Corollary 1. If $K$ is chosen such that $|K|<L$, then the columns of $M(\mathcal{V})$ are linearly dependent for any set $\mathcal{V}$ of examples, i.e., a unique weak solution does not exist.

While we are now aware of a lower bound for the size of $K$, it yet remains to prove that step (1) of our learning algorithm is, in fact, correct. This will be achieved by introducing the $\left(\left(2^{n}|K|\right) \times|T(n, L)|\right)$-matrix $M^{*}=M\left(\{0,1\}^{n} \times K\right)$, which clearly corresponds to the set of all possible examples, and showing that $M^{*}$ has full column rank $|T(n, L)|$ if $L \leq|K|$ holds. 
However, be careful not to misinterpret this finding, which is presented below in the form of Theorem 2. The fact that $M^{*}$ has full column rank $|T(n, L)|$ by no means implies that, eventually, this will also hold for $M(\mathcal{V})$ if only the corresponding set of observations $\mathcal{V}$ is large enough. In particular, the experimental results summarized in section 5 (see, e.g., table 10 show that there are cases in which the rank of $M(\mathcal{V})$ is always smaller than $|T(n, L)|$, even if $L \leq|K|$ is satisfied and $\mathcal{V}$ equals the set $\left\{\left(u, f_{l}(u)\right) \mid u \in\{0,1\}^{n}, l \in[L]\right\} \subseteq\{0,1\}^{n} \times K 5^{5}$ of all possible valid examples.

Still, as a counterpart of Theorem 11 the following theorem proves the possibility of existence of a unique weak solution for arbitrary parameters $n$ and $L$ satisfying $2 \leq L \leq n$. In other words, choosing $T(n, L)$ to be the set of basis polynomials does not necessarily lead to systems of linear equations which cannot be solved uniquely.

Theorem 2. Let $n$ and $L$ be arbitrarily fixed such that $2 \leq L \leq n$ holds. If $K$ satisfies $L \leq|K|$, then $M^{*}$ has full column $\operatorname{rank}|T(n, L)|$.

Proof: We denote by $\mathcal{Z}(n)$ the set of monomials $z_{0}^{d_{0}} \cdot \ldots \cdot z_{n}^{d_{n}}$, where $0 \leq$ $d_{i} \leq|K|-1$ for $i=0, \ldots, n$. Obviously, the total number of such monomials is $|\mathcal{Z}(n)|=|K|^{n+1}$. Let us recall the aforementioned fact that the relation $z^{1}=z^{|K|}$ holds for all $z \in K$ (including $0 \in K$ ). This straightforwardly implies that each monomial in the variables $z_{0}, \ldots, z_{n}$ is (as a function from $K^{n+1}$ to $K$ ) equivalent to a monomial in $\mathcal{Z}(n)$. Let $\mu_{J, j}$ denote the monomial $\mu_{J, j}=$ $z_{0}^{L-j} \prod_{r \in J} z_{r}$ for all $J \subseteq[n]$ and $j, 0 \leq j \leq L$. The following lemma can be easily verified:

Lemma 2.1. For all $J \subseteq[n], 1 \leq|J| \leq L$, and $j,|J| \leq j \leq L$, and examples $(u, w) \in\{0,1\}^{n} \times K$, it holds that $\mu_{J, j}(w, u)$ equals the coefficient in $M^{*}$ which has the coordinates $(u, w)$ and $t_{J, j}$.

For $i=1, \ldots,|K|$, we denote by $k_{i}$ the $i$-th element of the finite field $K$. Moreover, we suppose the convention that $0^{0}=1$ in $K$. Let $(u, w)$ be an example defined as above and keep in mind that we are treating the case $L \leq|K|$. It should be observed that the coefficients in the corresponding equation of type (2) are given by $w^{L-j}$, where $1 \leq j \leq L$. Thus, the set of possible exponents $\{L-j \mid 1 \leq j \leq L\}$ is bounded from above by $(L-1)<L \leq|K|$. It follows straightforwardly from Lemma 2.1 that the (distinct) columns of $M^{*}$ are columns of the matrix $W \otimes B^{\otimes n}$, where

$$
W=\left(k_{i}^{j}\right)_{i=1, \ldots,|K|, j=0, \ldots,|K|-1} \quad \text { and } \quad B=\left[\begin{array}{ll}
1 & 0 \\
1 & 1
\end{array}\right] .
$$

As $W$ and $B$ are regular, $W \otimes B^{\otimes n}$ is regular, too. This, in turn, implies that the columns of $M^{*}$ are linearly independent, thus proving Theorem 2.

${ }^{5}$ It can be seen easily that for random linear functions $f_{1}, \ldots, f_{L}$, the relation $\left\{\left(u, f_{l}(u)\right) \mid u \in\{0,1\}^{n}, l \in[L]\right\} \neq\{0,1\}^{n} \times K$ will always hold if $L<|K|$ and is still very likely to hold if $L=|K|$. 
We will see in section 4 that for $|K| \in O\left(d n L^{4}\right)$, the strong solution can be reconstructed from the weak solution in time $n^{O(L)}$ with error probability at most $d^{-1}$. Furthermore, section 5 will feature an experimental assessment of the number of random (valid) observations needed until $M(\mathcal{V})$ achieves full column rank $|T(n, L)|$ for various combinations of $n, L$, and $K$ (see table 2).

\section{On Computing a Strong Solution from the Unique Weak Solution}

Let $n, K, L$, and $f_{1}, \ldots, f_{L}$ be defined as before. Remember that the goal of our learning algorithm is to compute a strong solution fully characterized by the $L$ sets $\left\{\left(e^{i}, f_{l}\left(e^{i}\right)\right) \mid i \in[n]\right\}, l=1, \ldots, L$, where $e^{i}$ denotes the $i$-th element of the standard basis of $G F(2)^{n}$ and $f_{l}\left(e^{i}\right)=x_{i}^{l} \in K$. Obviously, this information can equivalently be expressed as a matrix $A \in K^{n \times L}$ defined by $A_{i, \cdot}=\left(x_{i}^{1}, \ldots, x_{i}^{L}\right)$ for all $i=1, \ldots, n$.

Hence, we have to solve the following problem: Compute the matrix $A \in K^{n \times L}$ from the information $t(A)$, where

$$
t(A)=\left(t_{J, j}(A)\right)_{J \subseteq[n], 1 \leq|J| \leq L,|J| \leq j \leq L}
$$

is the unique weak solution determined previously. But before we lay out how (and under which conditions) a strong solution $A$ can be found, we need to introduce the following two definitions along with an important theorem linking them:

Definition 2. Let for all vectors $x \in K^{L}$ the signature sgt $(x)$ of $x$ be defined as sgt $(x)=\left(|x|_{k}\right)_{k \in K}$, where $|x|_{k}$ denotes the number of components of $x$ which equal $k$.

Furthermore, consider the following new family of polynomials:

Definition 3. a) For all $L \geq 1$ and $j \geq 0$ let the simple symmetric polynomial $s_{j}$ over the variables $x_{1}, \ldots, x_{L}$ be defined by $s_{0}=1$ and

$$
s_{j}=\bigoplus_{S \subseteq[L],|S|=j} m_{S},
$$

where $m_{S}=\prod_{i \in S} x_{i}$ for all $S \subseteq[L]$. Moreover, we denote

$$
s(x)=\left(s_{0}(x), s_{1}(x), \ldots, s_{L}(x)\right)
$$

for all $x \in K^{L}$.

b) Let $n, L, 1 \leq L \leq n$, hold as well as $j, 0 \leq j \leq L$, and $J \subseteq[n]$. The symmetric polynomial $s_{J, j}: K^{n \times L} \longrightarrow K$ is defined by

$$
s_{J, j}(A)=s_{j}\left(\bigoplus_{i \in J} A_{i, \cdot}\right)
$$


for all matrices $A \in K^{n \times L}$. Moreover, we denote

$$
s_{J}(A)=\left(s_{J, 0}(A), \ldots, s_{J, L}(A)\right) .
$$

The concept of signatures introduced in Definition 2 and the family of simple symmetric polynomials described in Definition 3 will now be connected by the following theorem:

Theorem 3. For all $L \geq 1$ and $x, x^{\prime} \in K^{L}$ it holds that $s(x)=s\left(x^{\prime}\right)$ if and only if sgt $(x)=\operatorname{sgt}\left(x^{\prime}\right)$.

Proof: See appendix $\mathrm{A}$

Building on this result, we can then prove the following proposition, which is of vital importance for computing the strong solution $A$ on the basis of the corresponding weak solution $t(A)$ :

Theorem 4. Let $A \in K^{n \times L}$ and $t(A)$ be defined as before. For each subset $I \subseteq[n]$ of rows of $A$, the signature of the sum of these rows, i.e., sgt $\left(\bigoplus_{i \in I} A_{i, .}\right)$, can be computed by solely using information derived from $t(A)$, in particular, without knowing the underlying matrix A itself.

Proof: We first observe that the $s$-polynomials can be written as linear combinations of the $t$-polynomials. Trivially, the relation $t_{\{i\}, j}=s_{\{i\}, j}$ holds for all $i \in[n]$ and $j, 1 \leq j \leq L$. Moreover, for all $I \subseteq[n],|I|>1$, it holds that

$$
s_{I, j}=\bigoplus_{Q \subseteq I, 1 \leq|Q| \leq j}\left(\bigoplus_{g:[L] \longrightarrow[n],|\operatorname{dom}(g)|=j, i m(g)=Q} m_{g}\right)=\bigoplus_{Q \subseteq I, 1 \leq|Q| \leq j} t_{Q, j} .
$$

Note that for all $J \subseteq[n]$ and $j,|J| \leq j \leq L$, relation (5) implies

$$
t_{J, j}=s_{J, j} \oplus \bigoplus_{Q \subset J} t_{Q, j}
$$

By an inductive argument, we obtain from relation (6) that the converse is also true, i.e., the $t$-polynomials can be written as linear combinations of the $s$-polynomials.

We have seen so far that given $t(A)$, we are able to compute $s_{I, j}$ for all $j$, $1 \leq j \leq L$, and each subset $I \subseteq[n]$ of rows of $A$. Recall

$$
s_{I, j}(A)=s_{j}\left(\bigoplus_{i \in I} A_{i, \cdot}\right) \quad \text { and } \quad s_{I}(A)=\left(s_{I, 0}(A), \ldots, s_{I, L}(A)\right)
$$

from Definition 3 and let $x \in K^{L}$ be defined by $x=\bigoplus_{i \in I} A_{i, .}$ It can be easily seen that $s_{I}(A)=s(x)$ holds.

In conjunction with Theorem 3 this straightforwardly implies the validity of Theorem 4 
Naturally, it remains to assess the degree of usefulness of this information when it comes to reconstructing the strong solution $A \in K^{n \times L}$. In the following, we will prove that if $K$ is large enough, then with high probability, $A$ can be completely (up to column permutations) and efficiently derived from the signatures of all single rows of $A$ and the signatures of all sums of pairs of rows of $A$ :

Theorem 5. Let $K=G F\left(2^{a}\right)$ fulfill $|K| \geq \frac{1}{4} \cdot d \cdot n \cdot L^{4}$, i.e., $a \geq \log (n)+$ $\log (d)+4 \log (L)-2$. Then, for a random matrix $A \in_{U} K^{n \times L}$, the following is true with a probability of approximately at least $\left(1-\frac{1}{d}\right): A$ can be completely reconstructed from the signatures $\operatorname{sgt}\left(A_{i, \cdot}\right), 1 \leq i \leq n$, and $\operatorname{sgt}\left(A_{i, \cdot} \oplus A_{j, .}\right)$, $1 \leq i<j \leq n$.

\section{Proof: See appendix A.}

As we have seen now that, under certain conditions, it is possible to fully reconstruct the strong solution $A$ by solely resorting to information obtained from the weak solution $t(A)$, we can proceed to actually describe a conceivable approach for step (3) of the learning algorithm:

We choose a constant error parameter $d$ and an exponent $a$, i.e., $K=G F\left(2^{a}\right)$, in such a way that Theorem 5 can be applied. Note that $L \leq n$ and $|K| \in n^{O(1)}$. In a pre-computation, we generate two databases $D B_{1}$ and $D B_{2}$ of size $n^{O(L)}$. While $D B_{1}$ acts as a lookup table with regard to the one-to-one relation between $s(x)$ and $\operatorname{sgt}(x)$ for all $x \in K^{L}$, we use $D B_{2}$ to store all triples of signatures $S, S^{\prime}, \tilde{S}$ for which there is exactly one solution pair $x, y \in K^{L}$ fulfilling $\operatorname{sgt}(x)=S$ and $\operatorname{sgt}(y)=S^{\prime}$ as well as $\operatorname{sgt}(x \oplus y)=\tilde{S}$.

Given $t(A)$, i.e., the previously determined weak solution, we then compute $\operatorname{sgt}\left(A_{i, .}\right)$ for all $i, 1 \leq i \leq n$, and $\operatorname{sgt}\left(A_{i, .} \oplus A_{j, .}\right)$ for all $i, j, 1 \leq i<j \leq n$, in time $n^{O(1)}$ by using $D B_{1}$ and relation (5), which can be found in the proof of Theorem 4. According to Theorem 5. it is now possible to reconstruct $A$ by the help of database $D B_{2}$ with probability at least $1-\frac{1}{d}$.

\section{Experimental Results}

To showcase the detailed workings of our learning algorithm as well as to evaluate its efficiency at a practical level, we created a complete implementation using the computer algebra system Magma. In case of success, it takes approximately 90 seconds on standard PC hardware (Intel i7, $2.66 \mathrm{GHz}$, with $6 \mathrm{~GB}$ RAM) to compute the unique strong solution on the basis of a set of 10,000 randomly generated examples for $n=10, a=3$ (i.e., $K=G F\left(2^{a}\right)$ ), and $L=5$. Relating to this, we performed various simulations in order to assess the corresponding probabilities, which were already discussed in sections 3 and 4 from a theoretical point of view.

The experimental results summarized in table 1 clearly suggest that if $|K|$ is only slightly larger than the number $L$ of secret linear functions, then in all likelihood, $M(\mathcal{V})$ will eventually reach full (column) rank $|T(n, L)|$, thus allowing for the computation of a unique weak solution. Moreover, in accordance with 
Table 1. An estimate of the rank of $M(\mathcal{V})$ on the basis of all possible valid observations for up to 10,000 randomly generated instances of RandomSelect $(L, n, a)$. For each choice of parameters, $|T(n, L)|$ denotes number of columns of $M(\mathcal{V})$ as defined in section 2 and listed in table 2 .

\begin{tabular}{|c|c|c|c|c|c|c|c|}
\hline \multirow{2}{*}{\multicolumn{3}{|c|}{ Parameters }} & \multicolumn{5}{|c|}{ Performed Iterations } \\
\hline & & & \multicolumn{2}{|c|}{ Rank of $M(\mathcal{V})<|T(n, L)|$} & \multicolumn{2}{|c|}{ Rank of $M(\mathcal{V})=|T(n, L)|$} & \multirow{2}{*}{$\begin{array}{l}\text { Total } \\
\text { Number }\end{array}$} \\
\hline$n$ & $K$ & $L$ & Number & Ratio & Number & Ratio & \\
\hline 4 & $G F\left(2^{2}\right)$ & 2 & 37 & $0.37 \%$ & 9,963 & $99.63 \%$ & 10,000 \\
\hline 4 & $G F\left(2^{2}\right)$ & 3 & 823 & $8.23 \%$ & 9,177 & $91.77 \%$ & 10,000 \\
\hline 4 & $G F\left(2^{2}\right)$ & 4 & 7,588 & $75.88 \%$ & 2,412 & $24.12 \%$ & 10,000 \\
\hline 5 & $G F\left(2^{2}\right)$ & 4 & 4,556 & $45.56 \%$ & 5,444 & $54.44 \%$ & 10,000 \\
\hline 5 & $G F\left(2^{2}\right)$ & 5 & 10,000 & $100.00 \%$ & 0 & $0.00 \%$ & 10,000 \\
\hline 6 & $G F\left(2^{3}\right)$ & 4 & 0 & $0.00 \%$ & 1,000 & $100.00 \%$ & 1,000 \\
\hline 8 & $G F\left(2^{3}\right)$ & 4 & 0 & $0.00 \%$ & 1,000 & $100.00 \%$ & 1,000 \\
\hline 8 & $G F\left(2^{3}\right)$ & 6 & 0 & $0.00 \%$ & 100 & $100.00 \%$ & 100 \\
\hline 8 & $G F\left(2^{3}\right)$ & 7 & 0 & $0.00 \%$ & 100 & $100.00 \%$ & 100 \\
\hline 8 & $G F\left(2^{3}\right)$ & 8 & 0 & $0.00 \%$ & 100 & $100.00 \%$ & 100 \\
\hline 9 & $G F\left(2^{3}\right)$ & 8 & 0 & $0.00 \%$ & 10 & $100.00 \%$ & 10 \\
\hline 9 & $G F\left(2^{3}\right)$ & 9 & 10 & $100.00 \%$ & 0 & $0.00 \%$ & 10 \\
\hline
\end{tabular}

Corollary 1, the columns of $M(\mathcal{V})$ were always linearly dependent in the case of $n=5, K=G F\left(2^{2}\right)$ and $L=5$, i.e., $|K|=4<5=L$. A further analysis of the underlying data revealed in addition that, for arbitrary combinations of $n, K$, and $L$, the matrix $M(\mathcal{V})$ never reached full column rank if at least two of the corresponding $L$ random linear functions $f_{1}, \ldots, f_{L}$ were identical during an iteration of our experiments. Note that, on the basis of the current implementation, it was not possible to continue table 1 for larger parameter sizes because, e.g., in the case of $n=8, K=G F\left(2^{3}\right)$ and $L=7$, performing as few as 100 iterations already took more than 85 minutes on the previously described computer system.

Table 2 features additional statistical data with respect to the number of examples needed (in case of success) until the matrix $M(\mathcal{V})$ reaches full column rank $|T(n, L)|$. Please note that, in contrast to the experiments underlying table 1, such examples $\left(u, f_{l}(u)\right)$ are generated iteratively and independently choosing random pairs $u \in_{U}\{0,1\}^{n}$ and $l \in_{U}[L]$, i.e., they are not processed in their canonical order but observed randomly (and also repeatedly) to simulate a practical passive attack. While we have seen previously that for most choices of $n, K$ and $L$, the matrix $M(\mathcal{V})$ is highly likely to eventually reach full column rank, the experimental results summarized in table 2, most notably the observed $p$-quantiles, strongly suggest that our learning algorithm for RandomSelect $(L, n, a)$ will also be able to efficiently construct a corresponding LES which allows for computing a unique weak solution.

It remains to clear up the question, to what extent Theorem 5 reflects reality concerning the probability of a random $(n \times L)$-matrix over $K$ being sgt(2)identifiable (see Definitions 5]1 and 5]2 in the proof of Theorem 5), which is necessary and sufficient for the success of step (3) of our learning algorithm. Our 
Table 2. An estimate of the number of randomly generated examples $\left(u, f_{l}(u)\right)$ which have to be processed (in case of success) until the matrix $M(\mathcal{V})$ reaches full column rank $|T(n, L)|$. Given a probability $p$, we denote by $Q_{p}$ the $p$-quantile of the respective sample.

\begin{tabular}{|c|c|c|c|c|c|c|c|c|c|c|c|}
\hline \multicolumn{3}{|c|}{ Parameters } & \multirow[b]{2}{*}{$|T(n, L)|$} & \multicolumn{8}{|c|}{ Number of Random Examples until $\operatorname{Rank}(M(\mathcal{V}))=|T(n, L)|$} \\
\hline$n$ & $K$ & $L$ & & Avg. & Max. & Min. & $Q_{0.1}$ & $Q_{0.25}$ & $Q_{0.5}$ & $Q_{0.75}$ & $Q_{0.9}$ \\
\hline 4 & $G F\left(2^{2}\right)$ & 1 & 4 & 5.5 & 18 & 4 & 4 & 4 & 5 & 6 & 8 \\
\hline 4 & $G F\left(2^{2}\right)$ & 2 & 14 & 24.4 & 93 & 14 & 18 & 20 & 23 & 27 & 32 \\
\hline 4 & $G F\left(2^{2}\right)$ & 3 & 28 & 71.8 & 273 & 33 & 51 & 58 & 67 & 81 & 99 \\
\hline 4 & $G F\left(2^{2}\right)$ & 4 & 43 & 226.2 & 701 & 95 & 147 & 175 & 211 & 261 & 317 \\
\hline 5 & $G F\left(2^{2}\right)$ & 4 & 75 & 218.5 & 591 & 140 & 176 & 192 & 211 & 237 & 263 \\
\hline 6 & $G F\left(2^{3}\right)$ & 4 & 124 & 201.6 & 318 & 162 & 184 & 192 & 200 & 211 & 220 \\
\hline 8 & $G F\left(2^{3}\right)$ & 4 & 298 & 378.7 & 419 & 345 & 365 & 371 & 378 & 386 & 393 \\
\hline 8 & $G F\left(2^{3}\right)$ & 6 & 762 & 1401.6 & 1565 & 1302 & 1342 & 1364 & 1405 & 1427 & 1458 \\
\hline 8 & $G F\left(2^{3}\right)$ & 7 & 1016 & 2489.7 & 2731 & 2275 & 2369 & 2417 & 2477 & 2547 & 2645 \\
\hline 8 & $G F\left(2^{3}\right)$ & 8 & 1271 & 5255.3 & 7565 & 4302 & 4706 & 4931 & 5227 & 5557 & 5706 \\
\hline 9 & $G F\left(2^{3}\right)$ & 8 & 2295 & 6266.1 & 6553 & 6027 & 6078 & 6136 & 6199 & 6415 & 6504 \\
\hline
\end{tabular}

Table 3. An estimate of the ratio of $\operatorname{sgt}(2)$-identifiable $(n \times L)$-matrices over $K$

\begin{tabular}{|c|c|c|c|c|c|c|c|}
\hline \multirow{2}{*}{\multicolumn{3}{|c|}{ Parameters }} & \multicolumn{5}{|c|}{ Performed Iterations (i.e., randomly chosen $A \in U K^{n \times L}$ ) } \\
\hline & & & \multicolumn{2}{|c|}{$A$ not $\operatorname{sgt}(2)$-identifiable } & \multicolumn{2}{|c|}{$A$ was $s g t(2)$-identifiable } & \multirow{2}{*}{$\begin{array}{l}\text { Total } \\
\text { Number }\end{array}$} \\
\hline$n$ & $K$ & $L$ & Number & Ratio & Number & Ratio & \\
\hline 4 & $G F\left(2^{2}\right)$ & 2 & 0 & $0.00 \%$ & 10,000 & $100.00 \%$ & 10,000 \\
\hline 4 & $G F\left(2^{2}\right)$ & 3 & 69 & $0.69 \%$ & 9,931 & $99.31 \%$ & 10,000 \\
\hline 4 & $G F\left(2^{2}\right)$ & 4 & 343 & $3.43 \%$ & 9,657 & $96.57 \%$ & 10,000 \\
\hline 6 & $G F\left(2^{3}\right)$ & 4 & 0 & $0.00 \%$ & 10,000 & $100.00 \%$ & 10,000 \\
\hline 8 & $G F\left(2^{3}\right)$ & 4 & 0 & $0.00 \%$ & 10,000 & $100.00 \%$ & 10,000 \\
\hline 8 & $G F\left(2^{3}\right)$ & 6 & 0 & $0.00 \%$ & 1,000 & $100.00 \%$ & 1,000 \\
\hline 8 & $G F\left(2^{3}\right)$ & 7 & 0 & $0.00 \%$ & 1,000 & $100.00 \%$ & 1,000 \\
\hline 8 & $G F\left(2^{3}\right)$ & 8 & 0 & $0.00 \%$ & 100 & $100.00 \%$ & 100 \\
\hline 9 & $G F\left(2^{3}\right)$ & 8 & 0 & $0.00 \%$ & 100 & $100.00 \%$ & 100 \\
\hline
\end{tabular}

corresponding simulations yielded table 3, which immediately suggests that even for much smaller values of $|K|$ than those called for in Theorem 5, a strong solution $A \in_{U} K^{n \times L}$ can be completely reconstructed from the signatures $\operatorname{sgt}\left(A_{i, \cdot}\right)$, $1 \leq i \leq n$, and $\operatorname{sgt}\left(A_{i, .} \oplus A_{j, .}\right), 1 \leq i<j \leq n$. In conjunction with the experimental results concerning the rank of $M(\mathcal{V})$, this, in turn, implies that our learning algorithm will efficiently lead to success in the vast majority of cases.

\section{Discussion}

The running time of our learning algorithm for RandomSelect $(L, n, a)$ is dominated by the complexity of solving a system of linear equations with $|T(n, L)|$ unknowns. Our hardness conjecture is that this complexity also constitutes a 
lower bound to the complexity of RandomSelect $(L, n, a)$ itself, which would imply acceptable cryptographic security for parameter choices like $n=128$ and $L=8$ or $n=256$ and $L=6$. The experimental results summarized in the previous section clearly support this view. Consequently, employing the principle of random selection to design new symmetric lightweight authentication protocols might result in feasible alternatives to current HB-based cryptographic schemes.

A problem of independent interest is to determine the complexity of reconstructing an $s g t(r)$-identifiable matrix $A$ from the signatures of all sums of at most $r$ rows of $A$. Note that this problem is wedded to determining the complexity of RandomSelect $(L, n, a)$ with respect to an active learner, who is able to receive examples $(u, w)$ for inputs $u$ of his choice, where $w=f_{l}(u)$ and $l \in_{U}[L]$ is randomly chosen by the oracle. It is easy to see that such learners can efficiently compute $\operatorname{sgt}\left(f_{1}(u), \ldots, f_{L}(u)\right)$ by repeatedly asking for $u$. As the approach for reconstructing $A$ which was outlined in section 4 needs a data structure of size exponential in $L$, it would be interesting to know if there are corresponding algorithms of time and space costs polynomial in $L$.

From a theoretical point of view, another open problem is to determine the probability that a random $(n \times L)$-matrix over $\mathrm{K}$ is $s g t(r)$-identifiable for some $r, 2 \leq r \leq L$. Based on the results of our computer experiments, it appears more than likely that the lower bound derived in Theorem 5 is far from being in line with reality and that identifiable matrices occur with much higher probability for fields $K$ of significantly smaller size.

\section{References}

1. Armknecht, F., Krause, M.: Algebraic Attacks on Combiners with Memory. In: Boneh, D. (ed.) CRYPTO 2003. LNCS, vol. 2729, pp. 162-175. Springer, Heidelberg (2003)

2. Arora, S., Ge, R.: New algorithms for learning in presence of errors (submitted,

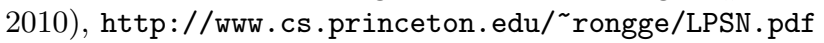

3. Blass, E.-O., Kurmus, A., Molva, R., Noubir, G., Shikfa, A.: The $F_{f}$-family of protocols for RFID-privacy and authentication. In: 5th Workshop on RFID Security, RFIDSec 2009 (2009)

4. Bogdanov, A., Knudsen, L.R., Leander, G., Paar, C., Poschmann, A., Robshaw, M.J.B., Seurin, Y., Vikkelsoe, C.: PRESENT: An Ultra-Lightweight Block Cipher. In: Paillier, P., Verbauwhede, I. (eds.) CHES 2007. LNCS, vol. 4727, pp. 450-466. Springer, Heidelberg (2007)

5. Bosma, W., Cannon, J., Playoust, C.: The Magma algebra system. I. The user language. J. Symbolic Comput. 24(3-4), 235-265 (1997)

6. Bringer, J., Chabanne, H.: Trusted-HB: A low cost version of $\mathrm{HB}^{+}$secure against a man-in-the-middle attack. IEEE Trans. Inform. Theor. 54, 4339-4342 (2008)

7. Cichoń, J., Klonowski, M., Kutyłowski, M.: Privacy Protection for RFID with Hidden Subset Identifiers. In: Indulska, J., Patterson, D.J., Rodden, T., Ott, M. (eds.) PERVASIVE 2008. LNCS, vol. 5013, pp. 298-314. Springer, Heidelberg (2008)

8. Courtois, N.: Fast Algebraic Attacks on Stream Ciphers with Linear Feedback. In: Boneh, D. (ed.) CRYPTO 2003. LNCS, vol. 2729, pp. 176-194. Springer, Heidelberg (2003) 
9. Courtois, N., Meier, W.: Algebraic Attacks on Stream Ciphers with Linear Feedback. In: Biham, E. (ed.) EUROCRYPT 2003. LNCS, vol. 2656, pp. 345-359. Springer, Heidelberg (2003)

10. De Cannière, C., Dunkelman, O., Knežević, M.: KATAN and KTANTAN - A Family of Small and Efficient Hardware-Oriented Block Ciphers. In: Clavier, C., Gaj, K. (eds.) CHES 2009. LNCS, vol. 5747, pp. 272-288. Springer, Heidelberg (2009)

11. Gołębiewski, Z., Majcher, K., Zagórski, F.: Attacks on CKK Family of RFID Authentication Protocols. In: Coudert, D., Simplot-Ryl, D., Stojmenovic, I. (eds.) ADHOC-NOW 2008. LNCS, vol. 5198, pp. 241-250. Springer, Heidelberg (2008)

12. Frumkin, D., Shamir, A.: Untrusted-HB: Security vulnerabilities of Trusted-HB. Cryptology ePrint Archive, Report 2009/044 (2009), http://eprint.iacr.org

13. Gilbert, H., Robshaw, M.J.B., Seurin, Y.: HB ${ }^{\#}$ Increasing the security and efficiency of $\mathrm{HB}^{+}$. In: Smart, N.P. (ed.) EUROCRYPT 2008. LNCS, vol. 4965, pp. 361-378. Springer, Heidelberg (2008)

14. Gilbert, H., Robshaw, M.J.B., Sibert, H.: Active attack against $\mathrm{HB}^{+}$: A provable secure lightweight authentication protocol. Electronic Letters 41, 1169-1170 (2005)

15. Goldreich, O., Levin, L.A.: A hard-core predicate for all one-way functions. In: Proceedings of the Twenty-First Annual ACM Symposium on Theory of Computing (STOC), pp. 25-32. ACM Press (1989)

16. Hopper, N.J., Blum, M.: Secure Human Identification Protocols. In: Boyd, C. (ed.) ASIACRYPT 2001. LNCS, vol. 2248, pp. 52-66. Springer, Heidelberg (2001)

17. Juels, A., Weis, S.A.: Authenticating Pervasive Devices with Human Protocols. In: Shoup, V. (ed.) CRYPTO 2005. LNCS, vol. 3621, pp. 293-308. Springer, Heidelberg (2005)

18. Krause, M., Stegemann, D.: More on the Security of Linear RFID Authentication Protocols. In: Jacobson Jr., M.J., Rijmen, V., Safavi-Naini, R. (eds.) SAC 2009. LNCS, vol. 5867, pp. 182-196. Springer, Heidelberg (2009)

19. Krause, M., Hamann, M.: The cryptographic power of random selection. Cryptology ePrint Archive, Report 2011/511 (2011), http://eprint.iacr.org/

20. Meier, W., Pasalic, E., Carlet, C.: Algebraic Attacks and Decomposition of Boolean Functions. In: Cachin, C., Camenisch, J.L. (eds.) EUROCRYPT 2004. LNCS, vol. 3027, pp. 474-491. Springer, Heidelberg (2004)

21. Ouafi, K., Overbeck, R., Vaudenay, S.: On the Security of HB\# against a Man-inthe-Middle Attack. In: Pieprzyk, J. (ed.) ASIACRYPT 2008. LNCS, vol. 5350, pp. 108-124. Springer, Heidelberg (2008)

22. Regev, O.: On lattices, learning with errors, random linear codes, and cryptography. In: Proceedings of the Thirty-Seventh Annual ACM Symposium on Theory of Computing (STOC), pp. 84-93. ACM Press (2005)

23. Courtois, N.T., Klimov, A.B., Patarin, J., Shamir, A.: Efficient Algorithms for Solving Overdefined Systems of Multivariate Polynomial Equations. In: Preneel, B. (ed.) EUROCRYPT 2000. LNCS, vol. 1807, pp. 392-407. Springer, Heidelberg (2000) 


\section{A The Proofs of Theorems 3 and 5}

Please refer to [19] for the full version of this paper including the proofs of Theorems 3 and 5 .

\section{B On Attacking the $(n, k, L)^{+}$-protocol by Solving RandomSelect $(L, n, a)$}

The following outline of an attack on the $(n, k, L)^{+}$-protocol by Krause and Stegemann [18] is meant to exemplify the immediate connection between the previously introduced learning problem RandomSelect $(L, n, a)$ and the security of this whole new class of lightweight authentication protocols. Similar to the basic communication mode described in the introduction, the $(n, k, L)^{+}$-protocol is based on $L n$-dimensional, injective linear functions $F_{1}, \ldots, F_{L}: G F(2)^{n} \longrightarrow$ $G F(2)^{n+k}$ (i.e., the secret key) and works as follows.

Each instance is initiated by the verifier Alice, who chooses a random vector $a \in_{U} G F(2)^{n / 2}$ and sends it to Bob, who then randomly (i.e., independently and uniformly) chooses $l \in_{U}[L]$ along with an additional value $b \in_{U} G F(2)^{n / 2}$, in order to compute his response $w=F_{l}(a, b)$. Finally, Alice accepts $w \in G F(2)^{n+k}$ if there is some $l \in[L]$ with $w \in V_{l}$ and the prefix of length $n / 2$ of $F_{l}^{-1}(w)$ equals $a$, where $V_{l}$ denotes the $n$-dimensional linear subspace of $G F(2)^{n+k}$ corresponding to the image of $F_{l}$.

This leads straightforwardly to a problem called Learning Unions of L Linear Subspaces (LULS), where an oracle holds the specifications of $L$ secret $n$ dimensional linear subspaces $V_{1}, \ldots, V_{L}$ of $G F(2)^{n+k}$, from which it randomly chooses examples $v \in_{U} V_{l}$ for $l \in_{U}[L]$ and sends them to the learner. Knowing only $n$ and $k$, he seeks to deduce the specifications of $V_{1}, \ldots, V_{L}$ from a sufficiently large set $\left\{w_{1}, \ldots, w_{s}\right\} \subseteq \bigcup_{l=1}^{L} V_{l}$ of such observations. It is easy to see that this corresponds to a passive key recovery attack against $(n, k, L)$-type protocols. Note that there is a number of exhaustive search strategies to solve this problem, e.g., the generic exponential time algorithm called search-for-a-basis heuristic, which was presented in the appendix of [18].

It should be noted that an attacker who is able to solve the LULS problem needs to perform additional steps to fully break the $(n, k, L)^{+}$-protocol as impersonating the prover requires to send responses $w \in G F(2)^{n+k}$ which not only fulfill $w \in \bigcup_{l=1}^{L} V_{l}$ but also depend on some random nonce $a \in G F(2)^{n / 2}$ provided by the verifier. However, having successfully obtained the specifications of the secret subspaces $V_{1}, \ldots, V_{L}$ allows in turn for generating a specification of the image of $F_{l}(a, \cdot)$ for each $l \in[L]$ by repeatedly sending an arbitrary but fixed (i.e., selected by the attacker) $a \in G F(2)^{n / 2}$ to the prover. Remember that, although the prover chooses a random $l \in_{U}[L]$ each time he computes a response $w$ based on some fixed $a$, an attacker who has determined $V_{1}, \ldots, V_{L}$ will know which subspace the vector $w$ actually belongs to. Krause and Stegemann pointed out that this strategy allows for efficiently constructing specifications of linear 
functions $G_{1}, \ldots, G_{L}: G F(2)^{n} \longrightarrow G F(2)^{n+k}$ and bijective linear functions $g_{1}, \ldots, g_{L}: G F(2)^{n / 2} \longrightarrow G F(2)^{n / 2}$ such that

$$
F_{l}(a, b)=G_{l}\left(a, g_{l}(b)\right)
$$

for all $l \in[L]$ and $a, b \in G F(2)^{n / 2}$ [18. Hence, the efficiently obtained specifications of the functions $\left(\left(G_{1}, \ldots, G_{L}\right),\left(g_{1}, \ldots, g_{L}\right)\right)$ are equivalent to the actual secret key $\left(F_{1}, \ldots, F_{L}\right)$. However, keep in mind that the running time of this attack is dominated by the effort needed to solve the LULS problem first and that Random Select $(L, n, a)$ in fact refers to a special case of the LULS problem, which assumes that the secret subspaces have the form

$$
V_{l}=\left\{\left(v, f_{l}(v)\right) \mid v \in G F(2)^{n}\right\} \subseteq G F(2)^{n+k}
$$

for all $l \in[L]$ and secret $G F(2)$-linear functions $f_{1}, \ldots, f_{L}: G F(2)^{n} \longrightarrow G F(2)^{k}$. This is true with probability $p(n) \approx 0.2887$ as, given an arbitrary $((n+k) \times n)$ matrix $A$ over $G F(2)$, the general case $V=\left\{A \circ v \mid v \in G F(2)^{n}\right\}$ can be written in the special form iff the first $n$ rows of $A$ are linearly independent (see, e.g., [11]).

In order to solve this special problem efficiently, we suggest the following approach, which makes use of our learning algorithm for RandomSelect $(L, n, a)$ and works by

- determining an appropriate number $a \in O(\log (n))$ which, w.l.o.g., divides $k$ (i.e., $k=\gamma \cdot a$ for some $\gamma \in \mathbb{N}$ ),

- identifying vectors $w \in\{0,1\}^{k}$ with vectors $w=\left(w_{1}, \ldots, w_{\gamma}\right) \in G F\left(2^{a}\right)^{\gamma}$ and functions $f:\{0,1\}^{n} \longrightarrow\{0,1\}^{k}$ with $\gamma$-tuples $\left(f^{1}, \ldots, f^{\gamma}\right)$ of component functions $f^{1}, \ldots, f^{\gamma}:\{0,1\}^{n} \longrightarrow G F\left(2^{a}\right)$ based on the following rule: $f^{i}(u)=w_{i}$ for all $i=1, \ldots, \gamma$ if and only if $f(u)=\left(w_{1}, \ldots, w_{\gamma}\right)$,

- learning $f_{1}, \ldots, f_{L}:\{0,1\}^{n} \longrightarrow\{0,1\}^{k}$ by learning each of the corresponding sets of component functions $f_{1}^{i}, \ldots, f_{L}^{i}:\{0,1\}^{n} \longrightarrow G F\left(2^{a}\right)$ in time $n^{O(L)}$ for $i=1, \ldots, \gamma$.

Clearly, for efficiency reasons, a should be as small as possible. However, in section 4 we show that $a$ needs to exceed a certain threshold, which can be bounded from above by $O(\log (n))$, to enable our learning algorithm to find a unique solution with high probability.

Please note that, throughout this paper, $a$ is assumed to be fixed as we develop a learning algorithm for sets of secret $G F(2)$-linear functions $f_{1}, \ldots, f_{L}$ : $\{0,1\}^{n} \longrightarrow K$, where $K=G F\left(2^{a}\right)$. In particular, for the sake of simplicity, we write $f_{1}, \ldots, f_{L}$ while actually referring to a set of component functions as explained above. 